\title{
Algorithms for time synchronization of wireless structural monitoring sensors
}

\author{
Ying Lei, Anne S. Kiremidjian*, ${ }^{*}$, K. Krishnan Nair, \\ Jerome P. Lynch and Kincho H. Law \\ John A. Blume Earthquake Engineering Center, Department of Civil and Environmental Engineering, \\ Stanford University, Stanford, CA94305, U.S.A.
}

\begin{abstract}
SUMMARY
Dense networks of wireless structural health monitoring systems can effectively remove the disadvantages associated with current wire-based sparse sensing systems. However, recorded data sets may have relative time-delays due to interference in radio transmission or inherent internal sensor clock errors. For structural system identification and damage detection purposes, sensor data require that they are time synchronized. The need for time synchronization of sensor data is illustrated through a series of tests on asynchronous data sets. Results from the identification of structural modal parameters show that frequencies and damping ratios are not influenced by the asynchronous data; however, the error in identifying structural mode shapes can be significant. The results from these tests are summarized in Appendix A.

The objective of this paper is to present algorithms for measurement data synchronization. Two algorithms are proposed for this purpose. The first algorithm is applicable when the input signal to a structure can be measured. The time-delay between an output measurement and the input is identified based on an ARX (auto-regressive model with exogenous input) model for the input-output pair recordings. The second algorithm can be used for a structure subject to ambient excitation, where the excitation cannot be measured. An ARMAV (auto-regressive moving average vector) model is constructed from two output signals and the time-delay between them is evaluated. The proposed algorithms are verified with simulation data and recorded seismic response data from multi-story buildings. The influence of noise on the time-delay estimates is also assessed. Copyright (c) 2004 John Wiley \& Sons, Ltd.
\end{abstract}

KEY WORDS: synchronization; time series analysis; wireless sensors; system identification; structural health monitoring

\footnotetext{
* Correspondence to: Anne S. Kiremidjian, Department of Civil and Environmental Engineering, Stanford University, Stanford, CA 94305, U.S.A.

†E-mail: ask@stanford.edu

Contract grant sponsor: National Science Foundation; contract grant number: CMS-0121841
}

Received 19 March 2003

Copyright (c) 2004 John Wiley \& Sons, Ltd.

Revised 30 October 2003 and 17 February 2004 Accepted 1 September 2004 


\section{INTRODUCTION}

There exists a clear need to monitor the health of large civil engineering structures over their operational lives and when subjected to extreme events such as earthquakes, hurricanes or blasts. Difficulties with installation and maintenance of current wired monitoring systems have led to the development of low-cost (less than $\$ 1000$ per sensing unit) wireless sensors for the health monitoring of civil structures [1-3]. Wireless sensors, however, may trigger at different times, thus data from sensors may not have the same initial time stamp. Furthermore, the transmission of the data may be delayed due to blockage of the signal or interference from other wireless devices that may be operating in the neighborhood of the system. Recent wireless sensor network designs [4] ensure that data loss is at a minimum; however, time delay in signal arrival at the data collection point cannot be prevented. In addition, there may be time-delays in the signal due to inherent clock errors. The objective of this paper is to present algorithms for synchronization of data that trigger at different times by the sensors. Two algorithms are presented for data synchronization. Synchronization due to blockage of the signal is currently under investigation and will be addressed in subsequent papers. The need for synchronization is illustrated through applications to structural modal parameter identification analysis. The results from these analyses are presented in Appendix A.

Until recently, recorded data (used to provide insight into the performance of structures) have come from cable connected sensors that use a common trigger. Structural modal parameters are estimated from these data using system identification algorithms [5-9]. Furthermore, methods for structural damage detection have been proposed based on changes of structural modal parameters, such as natural frequencies, damping ratios, mode shapes or mode shape curvatures [10-12]. With wireless systems, however, the trigger time can be different and the data from the various sensors may need to be synchronized in order to perform system identification and damage detection. In order to illustrate the need for synchronization, the influence of asynchronous data on identification results of structural modal parameters is investigated and presented in Appendix A. For this purpose, the ARX model [7-9] and the natural excitation technique (NExT) [13,14] are also summarized in Appendix A. The results from the application of these models show that modal frequencies and damping are not affected; however, mode shapes are significantly changed if the data are not synchronized. Thus, the focus of this paper is on the development of time synchronization algorithms.

Time synchronization of signals has been developed for other applications $[15,16]$, but not for wireless structural health monitoring purposes. In this paper, two algorithms are developed that can be used for synchronizing data in wireless sensor networks. The first algorithm can be used when the input to a structure is measured. The input signal serves as the reference signal, and each output signal is synchronized with the input signal. The second algorithm treats asynchronous output measurements from a structure under ambient excitation, for which the input signal is not measured and is not typically known. One of the output signals in this case is taken as the reference signal and all remaining signals are synchronized with the reference signal. The structure is assumed to behave linearly and the signals are stationary.

The time synchronization algorithms are tested with signals that have time delays that are either smaller or larger than the sampling rates. Several numerical examples of simulated and recorded seismic response data from multi-story buildings are used to demonstrate and verify the proposed algorithms for data synchronization. As the noise-to-signal ratio in the ambient 
vibration data may be quite high in many civil infrastructure applications, the effect of noise on the measured data and their synchronization is also considered herein.

\section{TIME SYNCHRONIZATION ALGORITHM FOR INPUT-OUTPUT PAIR OF RECORDINGS}

The first proposed algorithm assumes that the excitation and response signals are both measured. Thus, the input signal is selected as the reference signal. In general, the output signals recorded by wireless sensing units may have time-delay relative to the reference signal. The set of asynchronous input and output signals can be represented as follows:

$$
\begin{gathered}
\ldots, \ddot{x}_{g}\left(t_{m-1}\right), \ddot{x}_{g}\left(t_{m}\right), \ddot{x}_{g}\left(t_{m+1}\right), \ldots, \ddot{x}_{g}\left(t_{m+N}\right) \\
\ldots, \ddot{y}_{1}\left(t_{m-1}-\tau_{1}\right), \ddot{y}_{1}\left(t_{m}-\tau_{1}\right), \ddot{y}_{1}\left(t_{m+1}-\tau_{1}\right), \ldots, \ddot{y}_{1}\left(t_{m+N}-\tau_{1}\right) \\
\ldots, \ddot{y}_{2}\left(t_{m-1}-\tau_{2}\right), \ddot{y}_{2}\left(t_{m}-\tau_{2}\right), \ddot{y}_{2}\left(t_{m+1}-\tau_{2}\right), \ldots, \ddot{y}_{2}\left(t_{m+N}-\tau_{2}\right) \\
\vdots \\
\ldots, \ddot{y}_{M}\left(t_{m-1}-\tau_{M}\right), \ddot{y}_{M}\left(t_{m}-\tau_{M}\right), \ddot{y}_{M}\left(t_{m+1}-\tau_{M}\right), \ldots, \ddot{y}_{M}\left(t_{m+N}-\tau_{M}\right)
\end{gathered}
$$

where $\ddot{x}_{g}(t)$ is the acceleration input and $\ddot{y}_{j}(t)$ is the $j$-th acceleration output $(j=1,2, \ldots, M)$, and $M$ is the number of sensing units recording output signals. The objective is to synchronize all output data with the input record.

\subsection{Time synchronization algorithm}

The ARX model constructed from the above asynchronous data is

$$
\ddot{y}_{j}\left(t_{m}-\tau_{j}\right)+\sum_{i=1}^{n a} a_{i} \ddot{y}_{j}\left(t_{m}-\tau_{j}-i \Delta\right)=\sum_{i=0}^{n b} b_{i} \ddot{x}_{g}\left(t_{m}-n k^{\prime} \cdot \Delta-i \Delta\right)+\varepsilon_{j}^{\prime}\left(t_{m}\right)
$$

When $\tau_{j}$ is a multiple of the sampling interval $\Delta$, Equation (2) can be rewritten as

$$
\ddot{y}_{j}\left(t_{m}^{\prime}\right)+\sum_{i=1}^{n a} a_{i} \ddot{y}_{j}\left(t_{m}^{\prime}-i \Delta\right)=\sum_{i=0}^{n b} b_{i} \ddot{x}_{g}\left(t_{m}^{\prime}-n k^{\prime \prime} \cdot \Delta-i \Delta\right)+\varepsilon_{j}^{\prime}\left(t_{m}^{\prime}\right)
$$

where

$$
t_{m}^{\prime}=t_{m}-\tau_{j} \quad \text { and } \quad n k^{\prime \prime}=n k^{\prime}-\tau_{j} / \Delta
$$

To obtain synchronous data, Equation (3) has to be equivalent to Equation (A1) as defined in Appendix A. Comparing Equation (3) with Equation (A1), it can be observed that the only difference between the two equations is the value of the time-delay between the input and output signals, when

$$
n k^{\prime \prime}=n k \quad \text { and } \quad n k^{\prime}=n k+\tau_{j} / \Delta
$$


Thus, analytically, Equation (3) is equivalent to Equation (A1). Therefore, Equation (3) is the equation of an ARX model constructed from synchronous input-output data but with a time-delay value other than the $n k$ value given in Equation (A1). The model parameters $\left(a_{i}, b_{i}, n a, n b\right.$ and $\left.n k\right)$ in the ARX model given by Equation (A1) are obtained by minimizing the model error. Thus, the proposed synchronization algorithm is based on the minimization process as described in the following paragraphs.

The ARX model for the input $\ddot{x}_{g}$ and output $\ddot{y}_{j}$ at discrete-time point $t_{m}-\tau_{j}$ is expressed as

$$
\ddot{y}_{j}\left(t_{m}-\tau_{j}\right)+\sum_{i=1}^{n a} a_{i} \ddot{y}_{j}\left(t_{m}-\tau_{j}-i \Delta\right)=\sum_{i=0}^{n b} b_{i} \ddot{x}_{g}\left(t_{m}-\tau_{j}-n k \cdot \Delta-i \Delta\right)+\varepsilon_{j}\left(t_{m}-\tau_{j}\right)
$$

When $\tau_{j}>0$, Equation (6) can be written as

$$
\ddot{y}_{j}\left(t_{m}-\tau_{j}\right)+\sum_{i=1}^{n a} a_{i} \ddot{y}_{j}\left(t_{m}-\tau_{j}-i \Delta\right)=\sum_{k=0}^{n b} b_{i} \ddot{x}_{g}\left(t_{m}-\tau_{j}^{\prime}-n k^{\prime} \Delta-i \Delta\right)+\varepsilon_{j}\left(t_{m}-\tau_{j}\right)
$$

where

$$
n k^{\prime}=n k+\operatorname{fix}\left(\tau_{j} / \Delta\right) ; \quad \tau_{j}^{\prime}=\tau_{j}-\operatorname{fix}\left(\tau_{j} / \Delta\right) \Delta
$$

in which $\operatorname{fix}\left(\tau_{j} / \Delta\right)$ rounds off the element $\tau_{j} / \Delta$ to the nearest integer. The value of $n k^{\prime}$ takes into account the part of the time-delay which is a multiple of the sampling interval, whereas the value of $\tau_{j}^{\prime}$ includes the remainder part of the time-delay as shown in Equation (8).

Once the values of $\ddot{y}_{j}\left(t_{m}-\tau_{j}\right)$ and $\ddot{x}_{g}\left(t_{m}-\tau_{j}^{\prime}\right)$ are recorded, the model orders $n a, n b$ and the time-delay $n k^{\prime}$ are determined by observing the variation of $\sum \varepsilon_{j}^{2}\left(t_{m}-\tau_{j}\right)$ with all possible combinations of $n a, n b$ and $n k^{\prime}$. Using the Akaike's information theoretic criterion (AIC) or the Rissanen's minimum description length criterion (MDL), the optimal values of $n a, n b$ and $n k^{\prime}$ are chosen such that they give a minimum estimation error [7-9]. Therefore, the selected optimal ARX model, which is a realistic approximation of the actual system, has minimum model estimation error when the recorded input and output or two output measurements are synchronized.

However, data generated by wireless structural monitoring sensors are asynchronous as expressed by Equation (1). The value of $\tau_{j}^{\prime}$ is not known and the excitation values $\ddot{x}_{g}\left(t_{m}-\tau_{j}^{\prime}\right)$ are not recorded. To estimate the value of $\tau_{j}^{\prime}$, the values of the input signal at shifted time instances can be evaluated by a spline interpolation yielding the following set of input data

$$
\ddot{x}_{g}\left(t_{1}-\tau_{0}\right), \ddot{x}_{g}\left(t_{2}-\tau_{0}\right), \ldots, \ddot{x}_{g}\left(t_{N}-\tau_{0}\right)
$$

where $\tau_{0}$ is the value of the time shift. With different values of $\tau_{0}$, a set of shifted input signals is obtained. Then, each shifted input signal is paired with one of the output signals $\ddot{y}_{j}$ to construct an ARX model, which is given as

$$
\ddot{y}_{j}\left(t_{m}-\tau_{j}\right)+\sum_{k=1}^{n a} a_{k} \ddot{y}_{k}\left(t_{m}-\tau_{j}-k \Delta\right)=\sum_{k=0}^{n b} b_{k} \ddot{x}_{g}\left(t_{m}-\tau_{0}-n k^{\prime} \Delta-k \Delta\right)+\varepsilon_{j}\left(t_{m}-\tau_{j} \mid \tau_{0}\right)
$$


where $\varepsilon_{j}\left(t_{m}-\tau_{j} \mid \tau_{0}\right)$ is the prediction error of the model with a given value of $\tau_{0}$. Two vectors, $\beta$ and $\theta$, are defined as follows:

$$
\begin{aligned}
\beta\left(t_{m}, \tau_{0}\right)= & {\left[-\ddot{y}_{j}\left(t_{m}-\tau_{j}-\Delta\right), \ldots,-\ddot{y}_{j}\left(t_{m}-\tau_{j}-n_{a} \Delta\right),\right.} \\
& \left.\ddot{x}_{g}\left(t_{m}-\tau_{0}-n k^{\prime} \cdot \Delta-\Delta\right), \ldots, \ddot{x}_{g}\left(t_{m}-\tau_{0}-n k^{\prime} \cdot \Delta-n_{b} \Delta\right)\right]^{\mathrm{T}} \\
\boldsymbol{\theta}= & {\left[a_{1}, a_{2}, \ldots, a_{n a}, b_{0}, b_{1}, b_{2}, \ldots, b_{n b}\right]^{\mathrm{T}} }
\end{aligned}
$$

where the superscript $\mathrm{T}$ denotes a transpose. Equation (6) then can be rewritten as

$$
\ddot{y}_{j}\left(t_{m}-\tau_{j}\right)=\boldsymbol{\beta}^{\mathrm{T}}\left(t_{m}-\tau_{j}, \tau_{0}\right) \boldsymbol{\theta}+\varepsilon_{j}\left(t_{m}-\tau_{j} \mid \tau_{0}\right)
$$

For a given value of $\tau_{0}$, the total error $V\left(\boldsymbol{\theta} \mid \tau_{0}\right)$, defined as the sum of the squares of model errors at all measurement times, is given by

$$
V\left(\boldsymbol{\theta} \mid \tau_{0}\right)=\sum_{m=n n+1}^{N} \varepsilon_{j}^{2}\left(t_{m}-\tau_{j} \mid \tau_{0}\right)=\sum_{m=n n+1}^{N}\left[\ddot{y}_{j}\left(t_{m}-\tau_{j}\right)-\boldsymbol{\beta}^{\mathrm{T}}\left(t_{m}-\tau_{j} \mid \tau_{0}\right) \boldsymbol{\theta}\right]^{2}
$$

where $n n=\max (n a, n b)+n k^{\prime}$

The total error expressed by Equation (14) is minimized to obtain the coefficients of the ARX model $\hat{\boldsymbol{\theta}}[7,8]$. Then the synchronization error for different values of $\tau_{0}$ is taken to correspond to the point where $V\left(\boldsymbol{\theta} \mid \tau_{0}\right)$ is at its minimum given by

$$
e_{j}\left(\tau_{0}\right)=\min _{\boldsymbol{\theta}} V\left(\boldsymbol{\theta} \mid \tau_{0}\right)
$$

where ' $m i n$ ' is defined as the minimum value of the function.

Equation (10) is equivalent to Equation (7) when $\tau_{0}=\tau_{j}^{\prime}$ and thus, the optimal model parameters $n a, n b$ and $n k^{\prime}$ give the minimum estimation error. Therefore, $\tau_{0}$ is estimated by observing the variation of $e_{j}\left(\tau_{0}\right)$ for a range of $\tau_{0}$ values. The value of $\tau_{0}$ that minimizes $e_{j}\left(\tau_{0}\right)$ is taken as the estimated value of the time-delay in recording the output $\ddot{y}_{j}(t)$ relative to the input signal $\ddot{x}_{g}(t)$, i.e.

$$
\tau_{j}=\arg \left\{\min _{\tau_{0}} e_{j}\left(\tau_{0}\right)\right\}
$$

where 'arg' gives the argument of the function. Then, the corresponding shifted input signal, given by Equation (9), is synchronous with the output signal $\ddot{y}_{j}(t)$ when shifted by $\tau_{0}$ as defined by Equation (16).

In the case $\tau_{j}<0$ (non-causal shift), the starting data point of the input signal $\ddot{x}_{g}$ for the time synchronization should be chosen such that the output has a time-delay with respect to the input signal. Subsequently, the time synchronization reduces to the former case when $\tau_{j}>0$.

Similarly, all other output signals can be synchronized with the input signal using the above algorithm. Finally, structural mode shapes can be identified after all effective participating factors are estimated according to Equations (A6) and (A10) as discussed in Appendix A. It is important to note that the estimated value of $n k^{\prime}$ includes both the effects of $n k$ and $\tau_{j}$. Thus, once we have obtained the synchronized models (which are characterized by the model 
orders, $n a$ and $n b$, the coefficients $a_{i}, b_{i}$ and the time delay $n k^{\prime}$ ), the system identification can be performed successfully. These are further illustrated by the following examples.

\subsection{Example application}

2.2.1. A 3-story shear building under a sweep sine ground excitation. In order to illustrate the algorithm, a simple example is developed first. For this purpose, the 3-story shear building described by Clough and Penzien [17] is used. A sweep sine excitation is applied to the base of the structure. The ground excitation $\ddot{x}_{g}(t)$ is defined as:

$$
\ddot{x}_{g}(t)=\sin [0.3 \pi(2+t) t]
$$

The excitation has constant amplitude of $1 \mathrm{in} / \mathrm{s}^{2}$ with a linearly varying frequency of 0.3 to $6.3 \mathrm{~Hz}$ over $40 \mathrm{~s}$. The floor acceleration responses recorded by wireless sensing units at the first, second and third floors have time-delays of $2.002 \mathrm{~s}, 4.804 \mathrm{~s}$ and $6.005 \mathrm{~s}$, respectively, relative to the input signal. These asynchronous data are generated by numerical simulation. The sampling time is equal to $0.01 \mathrm{~s}$.

Each acceleration response datum is paired with the shifted input to apply the proposed algorithm. Based on the criteria of optimal model order for ARX models [7,8], the model parameters of the ARX model for the first, second and third floor acceleration response paired with the input are selected as $n a=n b=8$ and $n k^{\prime}=200, n k^{\prime}=480$ and $n k^{\prime}=600$, respectively. Figures $1(\mathrm{a})-(\mathrm{c})$ illustrate the variations of $e_{1}\left(\tau_{0}\right), e_{2}\left(\tau_{0}\right)$ and $e_{3}\left(\tau_{0}\right)$ for a range of $\tau_{0}$ values. From these figures, the value of $\tau_{j}^{\prime}$ can be evaluated by the minimizing arguments of $e_{j}\left(\tau_{0}\right)$ $(j=1,2,3)$ as described by Equation (16). Then the time-delays of the three floor acceleration response data relative to the ground excitation signal can be identified as described by Equation (8). These values are found to be identical to the time-delays introduced in the response signals, pointing to the accuracy of the synchronization.

After the output signal is synchronized with the input signal, the corresponding effective modal participation factor is evaluated according to Equation (A10). Structural mode shapes are identified after the effective participating factors have been estimated. These identified mode shapes are normalized with respect to the $3 \mathrm{rd}$ floor and are given as

$$
\hat{\boldsymbol{\Phi}}=\left[\begin{array}{ccc}
1.000 & 1.000 & 1.000 \\
0.649 & -0.607 & -2.542 \\
0.302 & -0.679 & 2.440
\end{array}\right]
$$

The true values of mode shapes are

$$
\boldsymbol{\Phi}=\left[\begin{array}{ccc}
1.000 & 1.000 & 1.000 \\
0.649 & -0.607 & -2.542 \\
0.302 & -0.679 & 2.439
\end{array}\right]
$$

Comparison of the true and identified values of mode shapes shows that the identification of the mode shapes is accurate (to the second or third decimal) using the data synchronized by the proposed algorithm. 

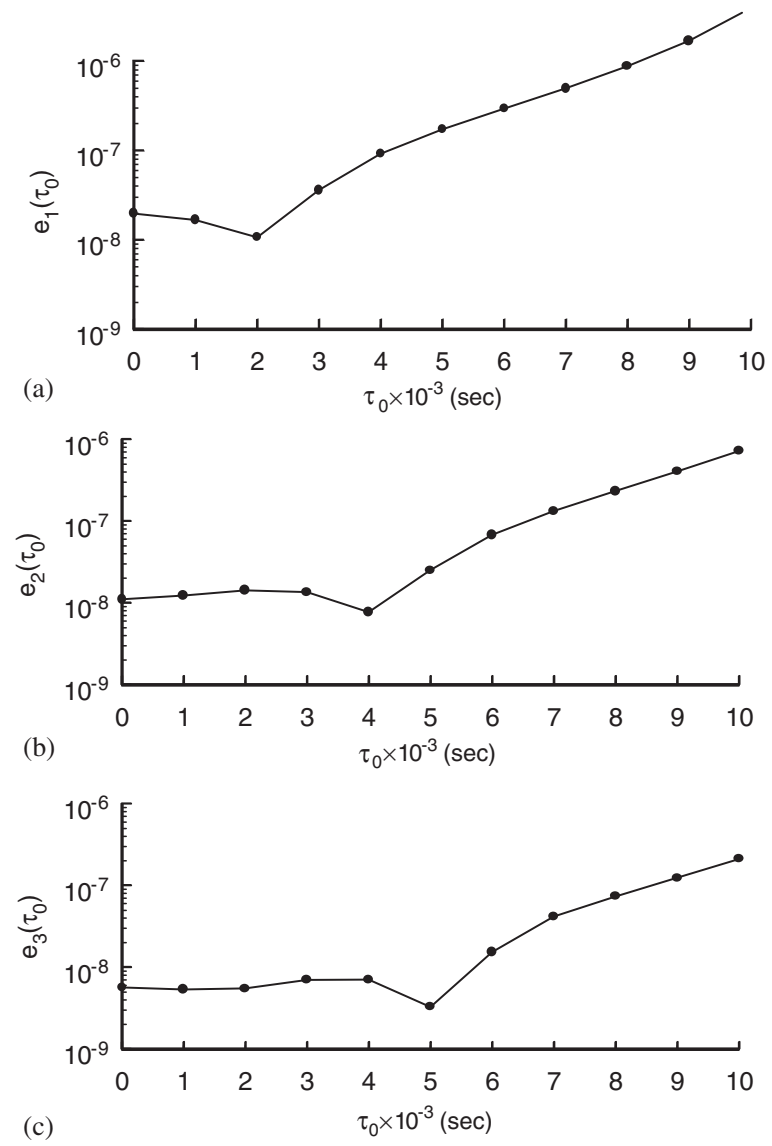

Figure 1. Variation of errors: (a) $e_{1}\left(\tau_{0}\right)$; (b) $e_{2}\left(\tau_{0}\right)$; and (c) $e_{3}\left(\tau_{0}\right)$ with $\tau_{0}$ under a sweep sine ground excitation.

2.2.2. The 3-story shear building under the El Centro earthquake excitation. In the second case study, the time synchronization algorithm is applied to the same 3-story building under the 1940 El Centro N-S earthquake loading with $P G A=0.3 \mathrm{~g}$. The recorded floor acceleration responses at the first, second and third floors are assumed to have time-delays of $5.004 \mathrm{~s}, 4.009 \mathrm{~s}$ and $7.006 \mathrm{~s}$, respectively, relative to the input signal. These are generated numerically with sampling interval equal to $0.02 \mathrm{~s}$. Using the Akaike's information theoretic criterion (AIC) or the Rissanen's minimum description length criterion (MDL), $n a, n b$ are selected as $n a=n b=8$ and $n k^{\prime}$ equal to 250, 200 and 350 in ARX models for first, second and third floor acceleration responses coupled with the excitation, respectively. Figures $2(\mathrm{a})-(\mathrm{c})$ illustrate the variations of $e_{1}\left(\tau_{0}\right), e_{2}\left(\tau_{0}\right)$ and $e_{3}\left(\tau_{0}\right)$ for a range of $\tau_{0}$ values. From these figures, the value of $\tau_{j}^{\prime}$ can be evaluated and the time-delays of the three floor acceleration response data relative to the ground excitation signal can be estimated by Equation (16). In order to comply with the stationarity assumption, only segments of the tail portion of the input and output signals can potentially be used for synchronization purposes where the structure is likely to be behaving 

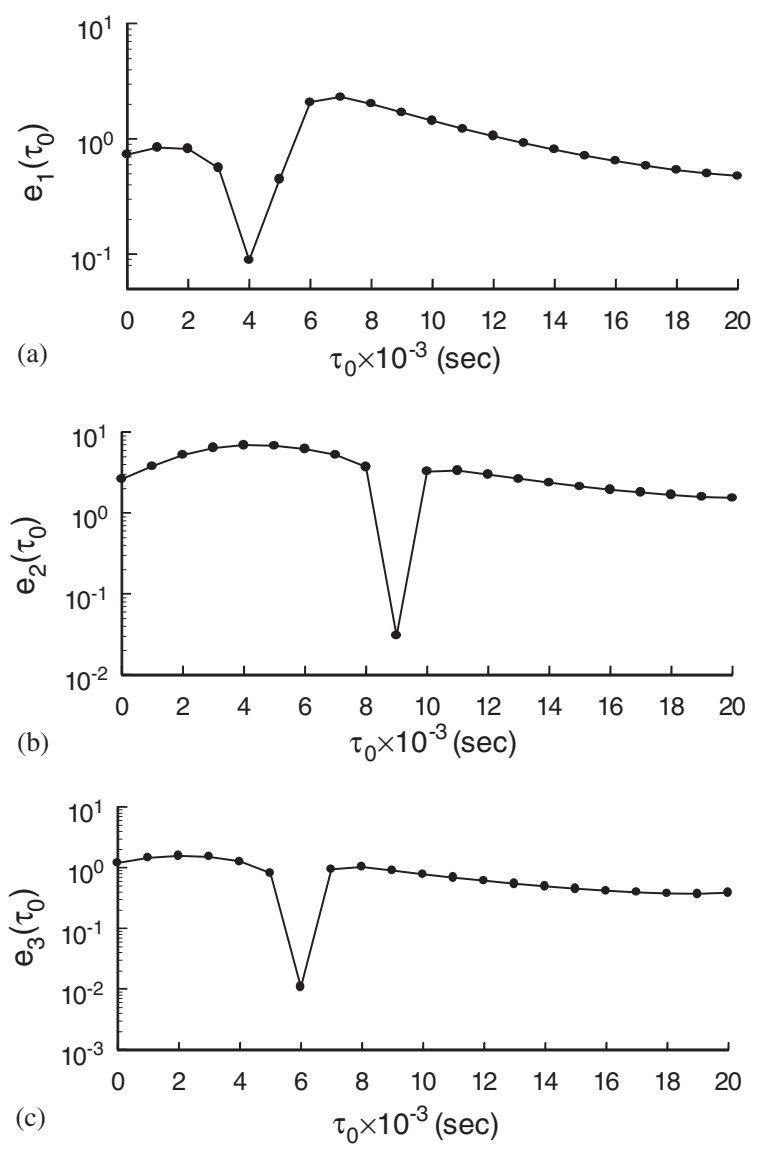

Figure 2. Variation of errors: (a) $e_{1}\left(\tau_{0}\right) ;(\mathrm{b}) e_{2}\left(\tau_{0}\right)$; and (c) $e_{3}\left(\tau_{0}\right)$ with $\tau_{0}$ under El Centro earthquake excitation.

linearly after the strong motion has subsided. This assumption is currently under investigation and results from the analyses will be reported in subsequent papers.

\subsubsection{Recorded accelerograms of an 18-story commercial building subject to the Loma} Prieta earthquake. The time synchronization algorithm is also demonstrated for the strongmotion accelerograms recorded in an 18-story commercial building in San Francisco subject to the 1989 Loma Prieta earthquake. The data were provided by the California Geological Survey's (CGS) Strong Motion Instrumentation Program (SMIP) (formerly Division of Mines and Geology, California Department of Conservation, ftp://ftp.consrv.ca.gov/pub/dmg/csmip/). The basement of the building is excited by one vertical and two horizontal ground motions. Under the condition that the three components of excitations recorded at the basement $\left(\ddot{x}_{1 g}, \ddot{x}_{2 g}, \ddot{x}_{3 g}\right)$ are synchronized, the above time-synchronization algorithm can be extended to a multi-input, single-output case. The ARX model for input-output data in Equation (10) is 
rewritten as:

$$
\begin{aligned}
\ddot{y}_{j}\left(t_{m}\right. & \left.-\tau_{j}\right)+\sum_{k=1}^{n a} a_{k} \ddot{y}_{k}\left(t_{m}-\tau_{j}-k \Delta\right)=\sum_{k=0}^{n b_{1}} b_{1 k} \ddot{x}_{1 g}\left(t_{m}-\tau_{0}-n k^{\prime} \cdot \Delta-k \Delta\right) \\
& +\sum_{k=0}^{n b_{2}} b_{2 k} \ddot{x}_{2 g}\left(t_{m}-\tau_{0}-n k^{\prime} \cdot \Delta-k \Delta\right) \\
& +\sum_{k=0}^{n b_{3}} b_{3 k} \ddot{x}_{3 g}\left(t_{m}-\tau_{0}-n k^{\prime} \cdot \Delta-k \Delta\right)+\varepsilon_{j}\left(t_{m}-\tau_{j} \mid \tau_{0}\right)
\end{aligned}
$$

where $n b_{1}, b_{1 k}, n b_{2}, b_{2 k}$, and $n b_{3}, b_{3 k}$ are orders and coefficients of the first, second and third exogenous input, respectively. A common time delay $n k^{\prime}$ for all three inputs is assumed here. Analogously, it can be shown that the relative time-delay value of $\tau_{j}$ can still be evaluated by minimizing $e_{j}\left(\tau_{0}\right)$ as described by Equation (16). To obtain asynchronous acceleration response data sets of the building, recorded accelerograms are artificially shifted to produce asynchronous data with constant time-delays. The structure is assumed to have sustained no damage, thus remaining in the linear range for the duration of the earthquake. The proposed time synchronization algorithm is then applied to treat the asynchronous data sets. In this numerical example, the original data-sampling interval is $0.02 \mathrm{~s}$, one recorded horizontal component of accelerograms at the 7th floor and another recorded horizontal component of accelerograms at the 12th floor are shifted so they have time-delays of $2.8 \mathrm{~s}$ and $3.4 \mathrm{~s}$ relative to the basement excitations, respectively. Using the Akaike's information theoretic criterion (AIC) or the Rissanen's minimum description length criterion (MDL), the optimal model values in the ARX model for the 7th floor response and basement excitation is $n a=16$, $n b_{1}=n b_{2}=n b_{3}=16, n k^{\prime}=140$, and they become $n a=18, n b_{1}=n b_{2}=n b_{3}=18, n k^{\prime}=170$ in the ARX model for the 12th floor response and basement excitation. These $n k^{\prime}$ values indicate that the 7th floor and 12th floor responses have time-delay values of $140 \times 0.02 \mathrm{~s}=2.8 \mathrm{~s}$ and $170 \times 0.02 \mathrm{~s}=3.4 \mathrm{~s}$, respectively, relative to the basement excitation. The results again show the accuracy of the time synchronization algorithm.

\section{TIME SYNCHRONIZATION ALGORITHM FOR OUTPUT RECORDINGS}

When a structure is subject to ambient excitation, the inputs to the structures cannot be measured. Typically only output signals are recorded by the wireless sensing units instrumented at different locations of the structure. One of the measured output signals $\ddot{y}_{\mathrm{r}}$ is chosen as the reference signal. The remaining measured acceleration responses have time-delays in recording data relative to the reference signal. Thus, the following asynchronous output data are recorded

$$
\begin{gathered}
\ldots, \ddot{y}_{1}\left(t_{m-1}-\tau_{1 \mathrm{r}}\right), \ddot{y}_{1}\left(t_{m}-\tau_{1 \mathrm{r}}\right), \ddot{y}_{1}\left(t_{m+1}-\tau_{1 \mathrm{r}}\right), \ldots, \ddot{y}_{1}\left(t_{m+N}-\tau_{1 \mathrm{r}}\right) \\
\ldots, \ddot{y}_{\mathrm{r}}\left(t_{m-1}\right), \ddot{y}_{\mathrm{r}}\left(t_{m}\right), \ddot{y}_{\mathrm{r}}\left(t_{m+1}\right), \ldots, \ddot{y}_{\mathrm{r}}\left(t_{m+N}\right) \\
\vdots \\
\ldots, \ddot{y}_{M}\left(t_{m-1}-\tau_{M \mathrm{r}}\right), \ddot{y}_{M}\left(t_{m}-\tau_{M \mathrm{r}}\right), \ddot{y}_{M}\left(t_{m+1}-\tau_{M \mathrm{r}}\right), \ldots, \ddot{y}_{M}\left(t_{m+N}-\tau_{M \mathrm{r}}\right)
\end{gathered}
$$


where $\tau_{j \mathrm{r}}$ is the unknown time-delay of the recorded output $\ddot{y}_{j}$ relative to the reference signal $\ddot{y}_{\mathrm{r}}$.

For structures under ambient excitation, auto-regressive moving average vector (ARMAV) models have been applied for system identification of structures [18-20]. These models only use a time series of output signals, without the requirement of excitation measurement. The excitation is assumed to be stationary Gaussian white noise. A time synchronization algorithm for output signals based on the ARMAV models is proposed.

\subsection{Time synchronization algorithm}

The values of the reference signal at shifted time instants are also evaluated by spline interpolation to yield the following data:

$$
\ddot{y}_{\mathrm{r}}\left(t_{1}-\tau_{0}\right), \ddot{y}_{\mathrm{r}}\left(t_{2}-\tau_{0}\right), \ldots, \ddot{y}_{\mathrm{r}}\left(t_{N}-\tau_{0}\right)
$$

where $\tau_{0}$ is the value of the time shift. With different values of $\tau_{0}$, a set of shifted reference signals is obtained.

An ARMAV model can be constructed from a shifted reference signal and another output $\ddot{y}_{j}$ by

$$
\ddot{\mathbf{y}}[n]=\sum_{k=1}^{p} \mathbf{a}_{k} \ddot{\mathbf{y}}[n-k]+\sum_{k=0}^{q} \mathbf{b}_{k} \mathbf{u}[n-k]+\mathbf{u}[n] ; \quad 1 \leqslant n \leqslant N
$$

where, $p$ and $q$ are the orders of the AR (auto-regressive) and MA (moving average) components respectively, $\mathbf{a}_{k}$ and $\mathbf{b}_{k}$ are $2 \times 2$ matrices of the AR and MA coefficients, and $N$ is the number of points in the records. $\ddot{\mathbf{y}}[n]=\left\{\ddot{y}_{\mathrm{r}}\left[n-\tau_{0}\right], \ddot{y}_{j}\left[n-\tau_{j \mathrm{r}}\right]\right\}^{\mathrm{T}}$ and $\mathbf{u}[n]=\{u[1, n], u[2, n]\}^{\mathrm{T}}$ are vectors of a pair of output signals and stationary zero-mean Gaussian white noise processes, respectively. The same ARMAV model in the state space can be rewritten as

$$
\begin{aligned}
& \underline{\ddot{\mathbf{y}}}[n]=\mathbf{A} \underline{\ddot{\mathbf{y}}}[n-1]+\mathbf{B} \underline{\mathbf{u}}[n] \\
& \ddot{\mathbf{y}}[n]=\mathbf{C} \underline{\ddot{y}}[n]
\end{aligned}
$$

where $\ddot{\mathbf{y}}[n]$ and $\underline{\mathbf{u}}[n]$ are vectors in the state space of dimension $2 p, \mathbf{A}$ and $\mathbf{B}$ are $2 p \times 2 p$ dimensional matrices containing the coefficients of AR and MA, respectively, and $\mathbf{C}$ is the observation matrix $[18,19]$.

Parameters of the ARMAV models are estimated by the prediction error method $[19,20]$. The vector $\boldsymbol{\theta}$ is defined as

$$
\boldsymbol{\theta}=\left[\mathbf{a}_{1}, \mathbf{a}_{2}, \ldots \mathbf{a}_{p}, \mathbf{b}_{0}, \mathbf{b}_{1}, \mathbf{b}_{2}, \ldots \mathbf{b}_{q}\right]^{\mathrm{T}}
$$

The prediction error vector $\varepsilon\left[n \mid \boldsymbol{\theta}, \tau_{0}\right]$ of the ARMAV model under a given value of $\tau_{0}$ can be expressed as

$$
\varepsilon\left[n \mid \boldsymbol{\theta}, \tau_{0}\right]=\ddot{\mathbf{y}}[n]-\hat{\mathbf{y}}[n]
$$

where $\ddot{\mathbf{y}}[n]$ is the vector of actual measured output values and $\hat{\mathbf{y}}[n]$ denotes the predicted value by the ARMAV model [20]. With a given value of $\tau_{0}, \hat{\boldsymbol{\theta}}$ can be obtained as the minimum 
point of a criterion function $V\left(\boldsymbol{\theta} \mid \tau_{0}\right)$. The criterion function $V\left(\boldsymbol{\theta} \mid \tau_{0}\right)$ is given as [19, 20]:

$$
V\left(\boldsymbol{\theta} \mid \tau_{0}\right)=\operatorname{det}\left\{\frac{1}{N} \sum_{n=1}^{N} \boldsymbol{\varepsilon}\left[n \mid \boldsymbol{\theta}, \tau_{0}\right] \boldsymbol{\varepsilon}\left[n \mid \boldsymbol{\theta}, \tau_{0}\right]^{\mathrm{T}}\right\}
$$

The minimum value of the criterion function under a given value of $\tau_{0}, w_{j \mathrm{r}}\left(\tau_{0}\right)$ is defined as:

$$
w_{j \mathrm{r}}\left(\tau_{0}\right)=\min _{\boldsymbol{\theta}} V\left(\boldsymbol{\theta} \mid \tau_{0}\right)
$$

The variation of $w_{j \mathrm{r}}\left(\tau_{0}\right)$ for a range of $\tau_{0}$ values is observed. The value of $\tau_{0}$, which gives the minimum value of $w_{j \mathrm{r}}\left(\tau_{0}\right)$, is taken as the estimated value of the time-delay in recording the selected output $\ddot{y}_{j}$ relative to the reference signal $\ddot{y}_{\mathrm{r}}$, i.e.

$$
\tau_{j \mathrm{r}}=\arg \left\{\min _{\tau_{0}} w_{j \mathrm{r}}\left(\tau_{0}\right)\right\}
$$

Subsequently, the shifted reference signal, given by Equation (20), with $\tau_{0}$ defined by Equation (27), is synchronous with the output $\ddot{y}_{j}(t)$. After obtaining the two synchronous output data, the corresponding modal elements of the structure $\boldsymbol{\Phi}$ can be extracted from the eigenvector matrix $\mathbf{L}$ of matrix $\mathbf{A}$ as [19]:

$$
\mathbf{\Phi}=\mathbf{C L}
$$

Alternately, other output measurements can be synchronized with the reference signal and structural mode shapes can be identified when there are as many measurements of the output as the number of degrees of freedom considered in the analysis.

\subsection{Example application}

A 4-story 2-bay by 2-bay shear building under ambient wind loading at each floor in the $y$-direction is considered to demonstrate the application of the proposed algorithm. This is one of the cases in the benchmark problem proposed by the ASCE Task Group on structural health monitoring [21] as shown in Figure 3. More information on the benchmark problem can be obtained from the website: http://wusceel.cive.wustl.edu/asce.shm/benchmarks.htm.

It is assumed that the measured acceleration data from the wireless sensing units at the first, second and third floors have time-delays of $2.6 \mathrm{~s}, 1.5 \mathrm{~s}$ and $0.9 \mathrm{~s}$, respectively, relative to the measured acceleration response of the fourth floor. These data are generated by the MATLAB program provided by the ASCE Task Group. The sampling interval of the output data is $0.01 \mathrm{~s}$. Ambient vibration measurements are likely to be affected by high noise. Hence, while performing system identification, the model should account for any noise in the data to obtain the correct modal parameters. In this paper, a band-limited Gaussian white noise process is used to model the noise and study its effects on the synchronization algorithm. The ratio of the root mean square (rms) value of the noise to the rms value of the fourth floor acceleration is $10 \%$ (noise to the largest signal ratio). The synchronization algorithm is applied to the noisy asynchronous data in order to estimate the accuracy of the time delay estimates. The acceleration response signal of the fourth floor is chosen as the reference signal. Figures $4(\mathrm{a})-(\mathrm{c})$ illustrate the variations of $w_{j 4}\left(\tau_{0}\right)(j=1,2,3)$ for a range of $\tau_{0}$ values. From 


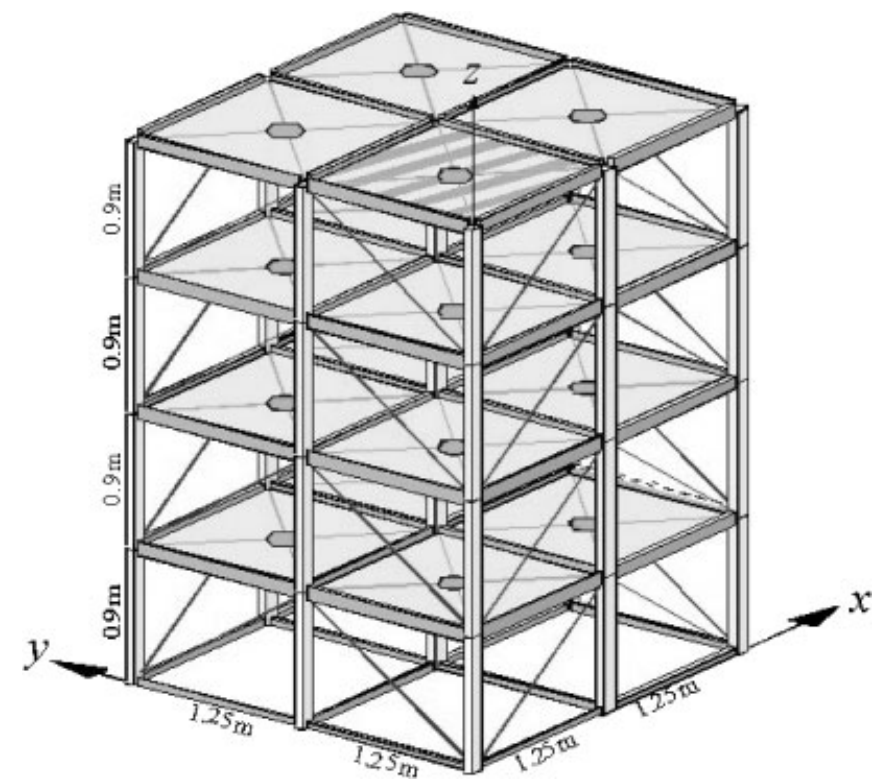

Figure 3. The benchmark building under ambient excitation in the $y$-direction [21].

the values of $\tau_{0}$, which produce the minimum values of $w_{j 4}\left(\tau_{0}\right)$, the time-delays in recording acceleration response data relative to the reference signal are evaluated accurately.

After obtaining the synchronous output data, the structural mode shapes in the $y$-direction can be identified using Equation (28). The identified mode shapes normalized with respect to the 4 th floor have the following amplitudes

$$
|\hat{\boldsymbol{\Phi}}|=\left[\begin{array}{llll}
1.000 & 1.000 & 1.000 & 1.000 \\
0.903 & 0.306 & 0.583 & 1.421 \\
0.687 & 0.708 & 0.829 & 1.238 \\
0.370 & 1.018 & 0.828 & 0.492
\end{array}\right]
$$

and the following phase angles (in degrees) are estimated as

$$
\operatorname{Phase}(\hat{\boldsymbol{\Phi}})=\left[\begin{array}{cccc}
0.000 & 0.000 & 0.000 & 0.000 \\
0.836 & 0.611 & 179.953 & 179.559 \\
1.230 & 179.318 & 179.712 & 2.520 \\
0.488 & 179.770 & 0.234 & 175.099
\end{array}\right]
$$



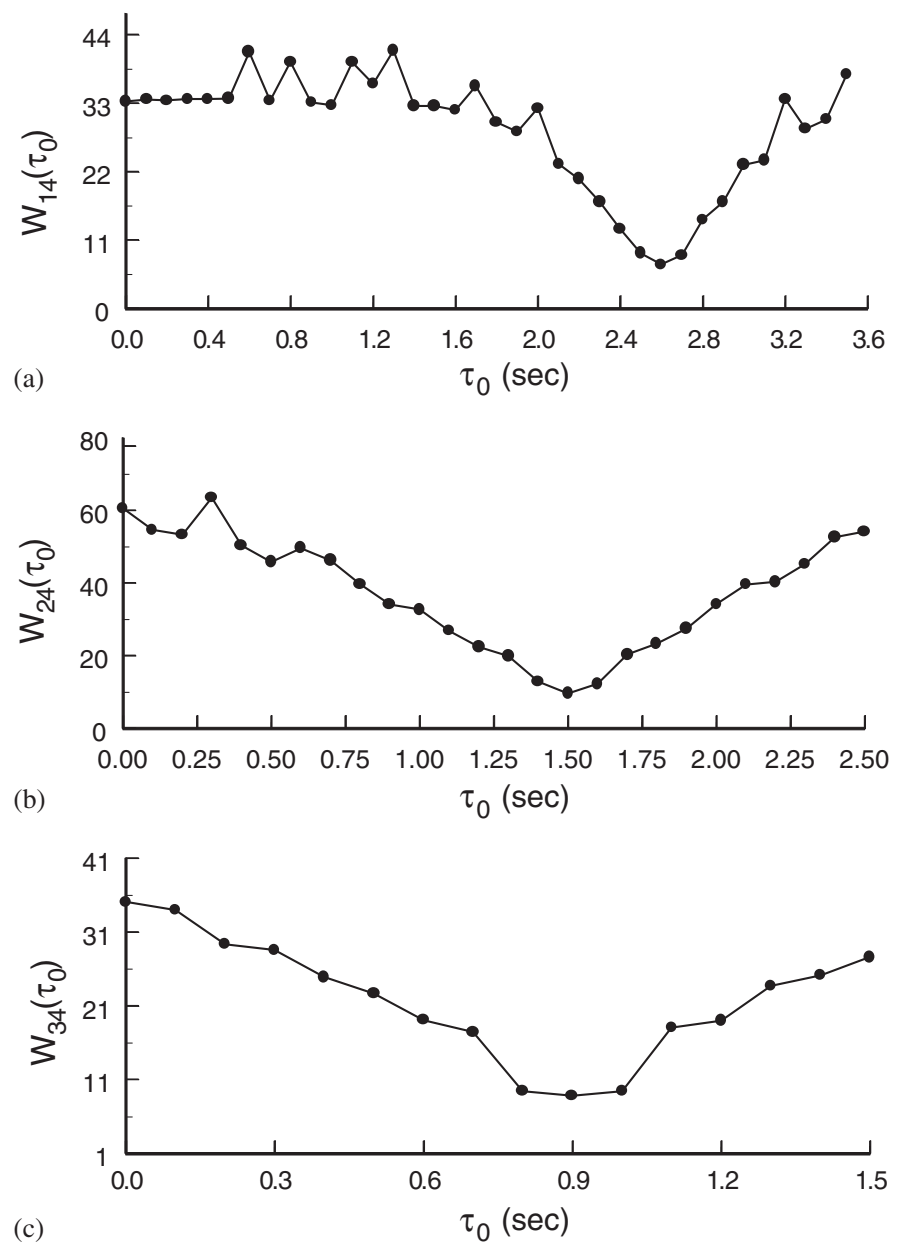

Figure 4. Variation of errors: (a) $w_{14}\left(\tau_{0}\right)$; (b) $w_{24}\left(\tau_{0}\right)$; and (c) $w_{34}\left(\tau_{0}\right)$ with $\tau_{0}$ for the benchmark problem.

The true values of mode shapes are

$$
\boldsymbol{\Phi}=\left[\begin{array}{cccc}
1.000 & 1.000 & 1.000 & 1.000 \\
0.907 & 0.313 & -0.573 & -1.425 \\
0.690 & -0.689 & -0.825 & 1.215 \\
0.379 & -0.998 & 0.825 & -0.463
\end{array}\right]
$$

Comparison of the true and identified values of mode shapes shows that the identification of the mode shapes is satisfactory using the data synchronized by the proposed algorithm. 


\section{CONCLUSIONS}

In this paper, two time synchronization algorithms are proposed to treat recorded asynchronous data for the purpose of accurate structural parameter identification and damage detection. Synchronization of signals is particularly important for the newly developed wireless sensor networks. The two algorithms estimate the time-delay by minimizing the model error associated with the ARX or ARMAV models. The first algorithm can be used when the input to a structure is measured. Output data are synchronized with the input data based on the ARX models for the input-output pairs. The algorithm is simple and its validity has been tested by several numerical examples of simulated and recorded seismic response data of buildings. Time-delays in recording output measurements relative to the measured ground input can be accurately evaluated as long as the numerical error due to interpolation of signals is small. The algorithm is illustrated with two examples.

The second algorithm can synchronize recorded outputs from structures under ambient excitation, where the input is unknown. It is based on the ARMAV model for a pair of output data, which requires more numerical effort in comparison to the first algorithm. The presence of noise in the ambient vibration data does not appear to affect the synchronization accuracy. Simulation data from the benchmark building proposed by the ASCE Task Group on structural health monitoring show that the second algorithm can also accurately synchronize output measurements. The preliminary analysis and the proposed algorithms are valid for stationary signals from linear systems. The effect of damage to structures and thus non-linear behavior is currently under investigation and will be reported in subsequent papers.

The influence of asynchronous data on the identification of structural modal parameters is investigated and the results are presented in Appendix A. It is shown that the identification of structural frequencies and damping ratios are not affected by the asynchronous data; however, the structural mode shapes are affected by the relative time-delay in recording the data. An analytical formulation is presented for the error in the identified structural mode shapes.

\section{APPENDIX A. EFFECTS OF TIME-DELAYS ON SYSTEM IDENTIFICATION}

\section{A.1. Structures with recorded single input}

A.1.1. ARX model from synchronous input-output data. When a structure is excited by a recorded ground excitation $\ddot{x}_{g}(t)$, auto-regressive models with exogenous input (ARX) have been used for system identification [7-9]. If the input $\ddot{x}_{g}(t)$ and the output signals $\ddot{y}_{j}(t)(j=1,2, \ldots, M)$ of the structure are recorded synchronously, an ARX model can be used to construct the input-output relationship in the discrete-time domain by the following equation

$$
\ddot{y}_{j}\left(t_{m}\right)+\sum_{i=1}^{n a} a_{i} \ddot{y}_{j}\left(t_{m}-i \Delta\right)=\sum_{i=0}^{n b} b_{i} \ddot{x}_{g}\left(t_{m}-n k \cdot \Delta-i \Delta\right)+\varepsilon_{j}\left(t_{m}\right) \quad \forall 1 \leqslant m \leqslant N
$$

where $\Delta$ is the sampling interval, $M$ is the number of sensing units in the recording output signals, $N$ is the number of points of recorded stationary data, $n a$ and $a_{i}$ are the order and coefficients of the AR terms (auto-regressive) respectively, $n b$ and $b_{i}$ are the order and coefficients of the exogenous input respectively, $n k \cdot \Delta$ is the time delay, in terms of the sampling interval $\Delta$, between the input $\ddot{x}_{g}(t)$ and output $\ddot{y}_{j}(t)$, and $\varepsilon_{j}\left(t_{m}\right)$ is the prediction 
error of the model. It is important to note that $n k$ would still be present in the case of synchronous data, which is a result of delay due to wave travel time and not due to delay to instrument recording.

The transfer function of the discrete system described by Equation (A1) is

$$
H_{j}(z)=\frac{\sum_{i=0}^{n b} b_{i} z^{-(i+n k)}}{1+\sum_{k=1}^{n a} a_{k} z^{-k}}
$$

where $z=\mathrm{e}^{s \Delta}$ and $s$ denotes the complex Laplace transform operator.

For $n b \leqslant n a$, the transfer function in Equation (A2) can be rewritten in the following form by using partial fraction expansions (if $n b>n a$, first a polynomial division is done and then a partial fraction expansion is made)

$$
H_{j}(z)=z^{-n k} \sum_{i=1}^{n a} \frac{r_{i}}{z-p_{i}}
$$

where $p_{i}$ is the pole of the transform function $H_{j}(z)$, which is determined by the roots of the denominator of $H_{j}(z)$, and $r_{i}$ denotes the residue of the transfer function $H_{j}(z)$ corresponding to the $i$-th pole $[7,8]$.

For a real system, the poles must be complex-conjugate pairs. To determine the contribution of each mode to the response, pairs of terms corresponding to pairs of complex-conjugate poles in Equation (A3) are combined together. Then, Equation (A3) is rewritten as:

$$
H_{j}(z)=z^{-n k} \sum_{i=1}^{n a / 2}\left[\frac{r_{i}}{\left(z-p_{i}\right)}+\frac{r_{i}^{*}}{\left(z-p_{i}^{*}\right)}\right]
$$

where, the superscript $*$ denotes the complex conjugate.

For a structure with proportional damping, the continuous frequency transfer function between the ground excitation and the acceleration output at point $j$ is well known [7,8]. If the signal has a time delay of $n k \cdot \Delta$ relative to the excitation, the transfer function can be derived as follows:

$$
H_{j}(s)=\sum_{i=1}^{n} \frac{\mathrm{e}^{-s n k \cdot \Delta} s^{2} \phi_{j i} \Gamma_{i}}{\left(s^{2}+2 \zeta_{i} \omega_{i} s+\omega_{i}^{2}\right)}=\mathrm{e}^{-s n k \cdot \Delta} \sum_{i=1}^{n} c_{j i}\left[\frac{\omega_{i}^{2} /\left(\lambda_{i}-\lambda_{i}^{*}\right)}{s-\lambda_{i}}+\frac{\omega_{i}^{2} /\left(\lambda_{i}^{*}-\lambda_{i}\right)}{s-\lambda_{i}^{*}}\right]
$$

where $n$ is the number of modes in the structure, $\omega_{i}=i$-th modal frequency, $\zeta_{i}=i$-th modal damping ratio, $\lambda_{i}=\omega_{i}\left(-\zeta_{i}+i \sqrt{1-\zeta_{i}^{2}}\right), \phi_{j i}=j$-th component of the $i$-th modal vector $\phi_{i}, \Gamma_{i}$ is the participating factor of the $i$-th mode and $c_{j i}$ is the effective participating factor of the $i$-th mode at point $j[22]$ defined as follows:

$$
c_{j i}=\phi_{j i} \Gamma_{i}=\phi_{j i} \frac{\boldsymbol{\phi}_{i}^{\mathrm{T}} \mathbf{M I}}{\boldsymbol{\phi}_{i}^{\mathrm{T}} \mathbf{M} \boldsymbol{\phi}_{i}^{\mathrm{T}}}
$$

where $\mathbf{M}$ is the mass matrix of the structure and $\mathbf{I}$ is a unit column vector.

Equations (A4) and (A5) are similar except that Equation (A4) is in the discrete-time domain while Equation (A5) is in the continuous-frequency domain. Based on the zero-orderhold equivalence technique $[7,23,24]$, the equivalent discrete-time transfer function of the 
continuous form can be derived as:

$$
H_{j}(z)=z^{-n k} \sum_{i=1}^{n a / 2} c_{j i}\left[\frac{\lambda_{i}\left(\mathrm{e}^{\lambda_{i} \Delta}-1\right) /\left(\lambda_{i}-\lambda_{i}^{*}\right)}{\left(z-\mathrm{e}^{\lambda_{i} \Delta}\right)}+\frac{\lambda_{i}^{*}\left(\mathrm{e}^{\lambda_{i}^{*} \Delta}-1\right) /\left(\lambda_{i}^{*}-\lambda_{i}\right)}{\left(z-\mathrm{e}^{\lambda_{i}^{*} \Delta}\right)}\right]
$$

By comparing Equation (A7) to Equation (A4), the following equalities are obtained

$$
p_{i}=\mathrm{e}^{\lambda_{i} \Delta} ; \quad r_{i}=c_{j i} \lambda_{i}\left(\mathrm{e}^{\lambda_{i} \Delta}-1\right) /\left(\lambda_{i}-\lambda_{i}^{*}\right)
$$

From Equation (A8), structural modal frequency $\omega_{i}$ and modal damping ratio $\zeta_{i}$ can be calculated as

$$
\omega_{i}=\frac{\left|\ln p_{i}\right|}{\Delta} ; \quad \zeta_{i}=\frac{-\ln \left|p_{i}\right|}{\left|\ln p_{i}\right|}
$$

where || denotes the modulus of the corresponding complex value. The effective participating factor $c_{j i}$ can be obtained from Equation (A8) and is given below

$$
c_{j i}=\frac{r_{i}\left(\lambda_{i}-\lambda_{i}^{*}\right)}{\lambda_{i}\left(p_{i}-1\right)}
$$

To determine the mode shapes of the structure, it is necessary to have as many measurements of the output as the degrees of freedom considered. Based on the definition of the effective participating factor $c_{j i}$ as shown in Equation (A6), the mode shapes of the structure can be identified.

A.1.2. ARX model from asynchronous input-output data. When the excitation to a structure is measured, the excitation signal is selected as a reference signal. As discussed before, output signals recorded by wireless sensing units might have time-delays relative to the reference signal resulting in asynchronous data. If the $j$-th acceleration output $\hat{\ddot{y}}_{j}(t)$ has a time-delay of $\tau_{j}$ relative to the input $\ddot{x}_{g}$, the ARX model in Equation (A1) is modified for the asynchronous output $\hat{\ddot{y}}_{j}(t)$ and input $\ddot{x}_{g}(t)$ as:

$$
\hat{\ddot{y}}_{j}\left(t_{m}+\tau_{j}\right)+\sum_{k=1}^{n a} a_{k} \hat{\ddot{y}}_{j}\left(t_{m}+\tau_{j}-k \Delta\right)=\sum_{k=0}^{n b} b_{k} \ddot{x}_{g}\left(t_{m}-n k \cdot \Delta-k \Delta\right)+\varepsilon_{j}\left(t_{m}\right)
$$

where $\hat{\ddot{y}}_{j}\left(t_{m}\right)=\ddot{y}_{j}\left(t_{m}-\tau_{j}\right)$.

The corresponding transfer function is

$$
\hat{H}_{j}(z)=\frac{z^{-\tau_{j} / \Delta} \sum_{i=0}^{n b} b_{i} z^{-i-n k}}{1+\sum_{i=1}^{n a} a_{k} z^{-i}}
$$

and it can be expanded into partial fractions analogous to Equation (A4) as [5]:

$$
\hat{H}_{j}(z)=\sum_{i=1}^{n a / 2}\left[\frac{z^{-\tau_{j} / \Delta} r_{i}}{\left(z-p_{i}\right)}+\frac{z^{-\tau_{j} / \Delta} r_{i}^{*}}{\left(z-p_{i}^{*}\right)}\right]
$$

By comparing Equation (A13) with Equation (A4), it is noted that the denominator of the transfer function is not influenced by the time-delay $\tau_{j}$. Thus, modal frequencies and damping ratios, determined from the roots of the denominator, $p_{i}$, as described by Equation (A9), are 
not influenced by the time-delay. However, the numerator of the transfer function depends on $\tau_{j}$. From Equations (A13) and (A10), the amplitude of the effective participating factor $\hat{c}_{j i}$ (identified from the asynchronous data) is given by

$$
\hat{c}_{j i}=c_{j i}\left|p_{i}\right|^{-\tau_{j} / \Delta}
$$

From Equation (A9), it can be derived that

$$
\left|p_{i}\right|^{-\tau_{j} / \Delta}=\mathrm{e}^{\zeta_{i} \omega_{i} \tau_{j}}
$$

The ratio of two components of a modal vector can be calculated from the ratio of the corresponding effective participating factors. Based on the definition of the effective participating factor $c_{j i}$ as shown by Equation (A6), the ratio of the two components in the $i$-th mode vector is changed as

$$
\frac{\hat{\phi}_{j i}}{\hat{\phi}_{\mathrm{r} i}}=\mathrm{e}^{\zeta_{i} \omega_{i} \tau_{j \mathrm{r}}} \frac{\phi_{j i}}{\phi_{\mathrm{r} i}} ; \quad \tau_{j \mathrm{r}}=\tau_{j}-\tau_{\mathrm{r}}
$$

where $\hat{\phi}_{j i}$ and $\hat{\phi}_{\mathrm{r} i}$ are the two components of the $i$-th modal vector $\hat{\boldsymbol{\phi}}_{i}$ identified from the asynchronous data, $\phi_{j i}$ and $\phi_{\mathrm{r} i}$ are the corresponding components identified from the synchronous data, and $\tau_{j \mathrm{r}}$ is the time-delay of the $j$-th output $\hat{\ddot{y}}_{j}(t)$ relative to the $r$-th output $\hat{\ddot{y}}_{\mathrm{r}}(t)$.

Thus, identification of structural frequencies and damping ratios is not influenced by the asynchronous data but the structural mode shapes are influenced by the relative time-delay in recording the data. Absolute quantities such as structural frequencies and damping ratios can be determined from a single output measured at a location that is not a node of structural modes. However, a relative quantity, such as a component of the modal vector depends on a pair of output measurements, where time synchronization of the two measurements is necessary.

Finally, the same results can be derived analogously to the cases where a structure is excited by a measured input at a point on the structure and/or the structure has non-proportional damping $[7,8]$.

\section{A.2. Structures under ambient excitation}

When a structure is subject to ambient excitation, the inputs to the structure are unknown. For stationary uncorrelated force inputs, it can be shown by the natural excitation technique (NExT) $[13,14]$ that the cross-correlation between two synchronous acceleration data $\ddot{y}_{\mathrm{r}}(t)$ and $\ddot{y}_{j}(t)$ has the following expression

$$
R_{\ddot{y}_{\mathrm{r}} \ddot{y}_{j}}(\tau)=E\left[\ddot{y}_{\mathrm{r}}(t) \ddot{y}_{j}(t+\tau)\right]=\sum_{i=1}^{n} \phi_{j i} A_{\mathrm{ri}} \mathrm{e}^{-\zeta_{i} \omega_{i} \tau} \sin \left(\omega_{d i} \tau+\theta_{j i}\right)
$$

where $A_{\mathrm{r} i}$ and $\theta_{j i}$ are constants $[13,14]$.

To extract modal parameters, $\ddot{y}_{\mathrm{r}}(t)$ is fixed. By treating the cross-correlation function in Equation (A16) as output from the free-vibration decay, various techniques [5, 25, 26] can be used to identify the modal frequency $\omega_{i}$, modal damping ratio $\zeta_{i}$ and ratios of the modal elements.

When wireless accelerometers are placed throughout the structure, one of the measured output signals $\ddot{y}_{\mathrm{r}}(t)$ is chosen as the reference signal. The remaining signals are presumed to 
have time-delays relative to the reference signal. If the $j$-th output recorded by the wireless sensing unit has a time-delay of $\tau_{j \mathrm{r}}$ relative to the reference signal, the cross-correlation function $\hat{R}_{j \mathrm{r}}(\tau)$ between these two asynchronous output measurements can be derived based on Equation (A16) as

$$
\begin{aligned}
\hat{R}_{\mathrm{r} j}(\tau) & =E\left[\hat{\ddot{y}}_{\mathrm{r}}(t) \hat{\ddot{y}}_{j}(t+\tau)\right]=E\left[\ddot{y}_{\mathrm{r}}(t) \ddot{y}_{j}\left(t-\tau_{j \mathrm{r}}+\tau\right)\right] \\
& =\sum_{i=1}^{n} \phi_{j i} A_{\mathrm{ri}} \mathrm{e}^{-\zeta_{i} \omega_{i}\left(-\tau_{j \mathrm{r}}+\tau\right)} \sin \left[\omega_{d i}\left(-\tau_{j \mathrm{r}}+\tau\right)+\theta_{j i}\right] \\
& =\sum_{i=1}^{n} \hat{\phi}_{j i} A_{\mathrm{ri}} \mathrm{e}^{-\zeta_{i} \omega_{i} \tau} \sin \left(\omega_{d i} \tau-\omega_{d i} \tau_{j \mathrm{r}}+\theta_{j i}\right)
\end{aligned}
$$

where $\hat{\ddot{y}}_{j}(t)$ is the $j$-th acceleration output recorded by the wireless sensing unit, and

$$
\hat{\phi}_{j i}=\phi_{j i} \mathrm{e}^{\zeta_{i} \omega_{i} \tau_{j \mathrm{r}}}
$$

Analogously, modal frequencies $\omega_{i}$, modal damping ratios $\zeta_{i}$ and ratios of the modal elements can be identified from the above cross-correlation function. By comparing Equation (A17) with Equation (A16), it is seen that the identification results of the modal frequency $\omega_{i}$ and modal damping ratio $\zeta_{i}$ are not influenced by the time-delay in the measurements, but the ratios of modal elements are influenced by the time-delays as expressed by Equation (A15).

\section{ACKNOWLEDGEMENTS}

This research is supported by the National Science Foundation through Grant No. CMS-0121841. We greatly appreciate their past support. We also thank the ASCE Task Group on Health Monitoring for providing the MATLAB codes to generate the data used in this study.

\section{REFERENCES}

1. Straser EG, Kiremidjian AS. Modular, wireless damage monitoring system for structures. Report No. 128, John A. Blume Earthquake Engineering Center, Department of Civil and Environmental Engineering, Stanford University, Stanford, CA. 1998.

2. Lynch JP, Law KH, Straser EG, Kiremidjian AS, Kenny TW. The development of a wireless modular health monitoring system for civil structures. Proceedings of the MCEER Mitigation of Earthquake Disaster by Advanced Technologies, Las Vegas, NV, U.S.A., 30-31 November 2000.

3. Lynch JP, Sundararajan A, Law KH, Kiremidjian AS. Embedding algorithms in a wireless structural monitoring system. Proceedings of the International Conference on Advances and New Challenges in Earthquake Engineering Research (ICANCEER02), Hong Kong, China, 19-20 August 2002.

4. Kottapalli VA, Kiremidjian AS, Lynch JP, Carryer ED, Kenny TW, Law KH, Lei Y. Two-tiered wireless sensor network architecture for structural health monitoring. Proceedings of SPIE's 10th International Symposium on Smart Structures and Materials, San Diego, CA, U.S.A., 2003.

5. Beck JL, May BS, Polidori DC. Determination of modal parameters from ambient vibration data for structural health monitoring. Proceedings of the First World Conference on Structural Control, Pasadena, CA, June 1994.

6. Ghanem R, Shinozuka M. Structural system identification I: Theory. Journal of Engineering Mechanics (ASCE) $1995 ; \mathbf{1 2 1}(2): 255-264$.

7. Safak E. Identification of linear structures using discrete-time filters. Journal of Structural Engineering (ASCE) 1991; 117(10):3064-3085. 
8. Safak E, Celebi M. Seismic response of Transamerica building. II: System identification. Journal of Structural Engineering (ASCE) 1991; 117(8):2405-2425.

9. Ljung L. System Identification-Theory for User. Prentice-Hall: Englewood Cliffs, NJ, 1987.

10. Doebling SW, Farrar CR, Prime MB, Shevitz DW. Damage identification and health monitoring of structural and mechanical systems from changes in their vibration characteristics: a literature review. Los Alamos National Laboratory Report, LA-13070-MS, Los Alamos National Laboratory, Los Alamos, NM, 1996.

11. Chang F-K (ed.). Proceedings of the 1st, 2nd and 3rd International Workshops on Structural Health Monitoring (1997, 1999 and 2001), Stanford University, Stanford, CA. CRC Press: New York.

12. Farrar CR, Doebling SW. An overview of modal-based damage identification methods. Proceedings of DAMAS Conference, Sheffield, U.K., June 1997.

13. James GH, Carne TG, Lauffer JP. The natural excitation technique for modal parameter extraction from operating wind turbines. SAND92-1666, UC-261, Sandia National Laboratories, 1993.

14. Farrar CR, James GH. System identification from ambient vibration measurements on a bridge. Journal of Sound and Vibration 1997; 205(1):1-18.

15. Cusani R. Performance of fast time delay estimators. IEEE Transactions on Acoustics, Speech, and Signal Processing 1989; 37(5):757-759.

16. Kozek M. Input-output synchronization with non-uniformly and asynchronously samples output data. Proceedings of the 38th Conference on Decision and Control. Phoenix, AZ, U.S.A., 1999.

17. Clough RW, Penzien J. Dynamics of Structures. McGraw-Hill: New York, 1993.

18. Bodeux JB, Golinval JC. Application of ARMAV models to the identification and damage detection of mechanical and civil engineering structures. Smart Materials and Structures 2001; 10:479-489.

19. Giorcelli E, Fasana A, Garibaldi L, Riva A. Modal Analysis and system identification using ARMAV models. Proceedings of IMAC 12, pp. 676-680, Honolulu, HI, 1994.

20. Piombo B, Giorcelli E, Garibaldi L, Fasaba A. Structure identification using ARMAV models. Proceedings of IMAC 11, pp. 588-592, Orlando, FL, 1993.

21. Johnson EA, Lam HF, Katafygiotis LS, Beck JL. A benchmark problem for structural health monitoring and damage detection. Proceedings of the 14th Engineering Mechanics Conference, Austin, TX, U.S.A., 2000, CD version.

22. Beck JL. Determining models of structures from earthquake records. Technical Report EERL 78-01, Earthquake Engineering Research Laboratory, California Institute of Technology, Pasadena, CA, 1978.

23. Åström KJ, Wittenmark B. Computer-Controlled Systems: Theory and Design. Prentice-Hall, pp. 48-52, 1990.

24. Franklin GF, Powell JD, Workman ML. Digital Control of Dynamic Systems (2nd edn). Addison-Wesley, 1990.

25. Dyke SJ, Caicedo JM, Johnson EA. Monitoring of a benchmark structure for damage identification. Proceedings of the Engineering Mechanics Specialty Conference, Austin, Texas, 21-24 May 2000.

26. Yang JN, Lei Y, Pan S, Huang N. System identification of linear structures based on Hilbert-Huang spectral analysis. Part 1: normal modes. Earthquake Engineering and Structural Dynamics 2003; 32(9):1443-1467. 